\title{
Respuestas de las mujeres shuar al conflicto socioambiental en el área del Proyecto Mirador (Ecuador)
}

\author{
Ana Dolores Verdú Delgado ${ }^{1}$
}

Recibido: 18 de mayo de 2016 / Aceptado: 23 de marzo de 2017

Resumen: El Proyecto Mirador es un proyecto de minería a gran escala situado en la Cordillera del Cóndor que planea iniciar aproximadamente en 2018 la extracción de unas 60.000 toneladas de roca al día. La magnitud del impacto socio-ambiental que produce esta actividad se debe a su vez a que se da en un área habitada en parte por la nacionalidad indígena shuar, que disfruta del derecho constitucional a conservar sus territorios ancestrales.

En este texto analizo cuáles son los impactos que tiene la minería a gran escala sobre la población femenina shuar y cuál es su percepción en torno al conflicto que plantea. Los datos que manejo mantienen coherencia con el discurso ecofeminista centrado en la particularidad de las mujeres rurales de los países del "Sur" en relación con la naturaleza y el desarrollo.

Palabras clave: Ecofeminismo; mujeres indígenas; extractivismo; Amazonía.

\section{[en] Shuar Women's Responses to Socio-Environmental Conflict in the Area of the Mirador Project (Ecuador)}

\begin{abstract}
The Mirador Project is a large-scale mining project located in the Cordillera del Cóndor that plans to extract 60,000 metric tons of rock daily beginning in 2018 . The severity of socio-environmental impacts resulting from the Mirador Project are amplified due to the fact that the project takes place in an area largely inhabited by the indigenous Shuar who have constitutional rights to maintain control over their ancestral territories.

In this paper I analyze the impact that large-scale mining has on the Shuar female population by examining their perception of the conflict in question. Data is analyzed within the ecofeminist discourse focusing on the particularity of rural women in the global South in relation to nature and development. Keywords: Ecofeminism; indigenous women; extractivism; Amazonia.
\end{abstract}

Sumario. 1. Cambio social y conflicto en las sociedades shuar de la Amazonía ecuatoriana. 2. Mujeres shuar, conflicto y extractivismo. 3. Metodología. 4. El papel de las mujeres shuar dentro del conflicto socioambiental asociado al Proyecto Mirador. 4.1. Importancia del sistema de valores shuar en el discurso femenino en relación al conflicto. 4.2. Cambios en las relaciones de género. 4.3. Pérdida de la responsabilidad femenina sobre la reproducción cultural en el proceso de aculturación. 4.4. Las mujeres shuar ante un futuro amenazado por la minería a gran escala 5. Conclusiones. 6. Referencias bibliográficas.

Cómo citar: Verdú Delgado, A. M. (2017). Respuestas de las mujeres shuar al conflicto socioambiental en el área del Proyecto Mirador (Ecuador), en Revista de Antropología Social 26(1), 9-30.

1 Antropóloga Social y Cultural y Doctora en Estudios de Género, Universidad Técnica Particular de Loja (Ecuador), coordinadora del grupo de investigación "Enfoques Sociales del Desarrollo: Género e Interculturalidad" y colaboradora del Observatorio de Conflictos Socioambientales.

adverdu@utpl.edu.ec 


\section{Cambio social y conflicto en las sociedades shuar de la Amazonía ecuatoriana}

Los shuar son una de las once nacionalidades indígenas que habitan la Amazonía ecuatoriana, con una población estimada de unas 110.000 personas, según el Consejo de Desarrollo de las Nacionalidades y Pueblos del Ecuador (CODENPE) (Giz, 2011: 3), de las cuales más de 40.000 viven en Ecuador (Lu, Bilsborrow y Oña, 2012: 75). La nación Shuar se reconoce históricamente por ser la única del país que no se ha dejado someter por ningún pueblo invasor (Costales y Costales, 2006: 7), habiendo participado en duros enfrentamientos con los españoles durante los siglos XVI-XVIII (Barriga, 1986: 13-14).

Sus primeros contactos estables con foráneos en Ecuador son de hecho ya a finales del siglo XIX, con los misioneros que se instalan definitivamente en Gualaquiza, Macas, Napo, Canelos y Méndez (Barriga, 1986: 33), y a comienzos del siglo XX, con los misioneros católicos de la orden Salesiana que establecen misiones en las faldas de los Andes con el objetivo de convertir a los shuar al cristianismo (Lu, Bilsborrow y Oña, 2012: 75). Sucesivamente se asientan en sus territorios familias de colonos mestizos (especialmente entre los años 1920 y 1960), lo que se intensifica después de las reformas agrarias de Ecuador (años 1964-1973 y 1974-1980) (Ibidem: 75). Estos acontecimientos suponen el inicio de los conflictos que los shuar enfrentan en torno al territorio ${ }^{2}$, así como cambios en su sistema de asentamientos. Las sociedades shuar abandonan en esta época el estilo de vida en hogares dispersos para formar grupos de casas o "centros" (de entre 3.000 y 6.000 hectáreas) (Ibidem: 75), lo que también conlleva cambios en su sistema de producción: Tradicionalmente este pueblo realizaba actividades agrícolas y de crianza de animales domésticos y practicaba la caza y la pesca, orientadas al auto-consumo. Posteriormente, como los colonos, los shuar empiezan a limpiar la selva para el cultivo y a utilizar ganado (Ibidem: 76), cambios que se acentúan con la intensificación de actividades extractivas en sus territorios o entorno más próximo. En la actualidad, la fuente de ingresos de la mayor parte de la población shuar proviene principalmente de la venta directa de los productos de sus cultivos, de animales domésticos y de madera, y por otro lado, de empresas extractivas, dentro de las cuales realizan trabajos generalmente temporales y de escasa cualificación (Lu, Bilsborrow y Oña, 2012: 83).

Las importantes transformaciones sufridas por las sociedades shuar en las últimas décadas han puesto de manifiesto la centralidad del territorio en la manifestación del conflicto en un contexto en el que conviven y/o coexisten diferentes formas de entender la propiedad y la explotación de la naturaleza. De ahí que desde la creación de las organizaciones políticas de carácter étnico en la década de 1960 (Santos, 1996: 20), este problema haya estado presente de forma constante. Tanto la Federación de Centros Shuar (desde mediados de los años 60) como la CONAIE (Confederación de Nacionalidades Indígenas del Ecuador) han tenido como eje de sus reivindicaciones la lucha por el territorio. Estas luchas dan como resultado el reconocimiento de derechos colectivos para las poblaciones indígenas en la Constitución del Ecuador desde 1998. En la actualidad, la Constitución de la República del Ecuador 2008 reconoce el dere-

Frente al sistema shuar en el que no existe la propiedad privada de la tierra, se impuso en esta época un modelo de propiedad que permitía al colono a los colonos apropiarse de territorio mediante su trabajo agrícola y ganadero, lo que aceleró la reorganización del sistema de asentamiento de los shuar, respaldados por los salesianos (Lu, Bilsborrow y Oña, 2012: 75). 
cho de los pueblos y nacionalidades indígenas a mantener la posesión de sus territorios ancestrales mediante su adjudicación gratuita (artículo 57), así como el de constituir circunscripciones territoriales para la preservación de su cultura (artículo 60).

No obstante, al mismo tiempo que se reconocen los derechos territoriales indígenas, aumenta también la extensión de las áreas protegidas y, por otro lado, de las concesiones a empresas extractivas (Espinosa, 1998: 30) que transforman los patrones de uso de la tierra y agotan los recursos naturales, sin dejar apenas beneficios en la región (1998: 29). Como además, su explotación por el Estado (siendo delegada a terceras partes) conlleva la expulsión y expropiación de territorio de comunidades indígenas y campesinas, en 1985 se introduce el concepto de "servidumbre", con el que se reconoce la ocupación legítima de la empresa a la que se otorga la concesión sobre los predios superficiales (Colectivo de Investigación y Acción Psicosocial Ecuador, 2017: 52). La apuesta de la administración presidida por Rafael Correa por la minería a gran escala, según Gudynas (2009), siendo coherente con las acciones promovidas por los "nuevos gobiernos progresistas" o de "izquierda" en toda América Latina, contiene una especial contradicción. En la legislación ecuatoriana, al mismo tiempo que se recoge el derecho de los pueblos a la consulta previa, libre e informada sobre "programas de prospección, explotación y comercialización de recursos no renovables que se encuentren en sus tierras y que puedan afectarles ambiental o culturalmente" (artículo 57 de la Constitución de 2008), los bienes del subsuelo, del cobre y de otros minerales son considerados de propiedad nacional.

En consecuencia, la cuestión territorial sigue constituyendo un factor de enorme trascendencia en la percepción de conflictos por parte del pueblo shuar, que a pesar de disfrutar del derecho constitucional a conservar sus territorios ancestrales, en la realidad se enfrenta a la contaminación en las áreas invadidas por el extractivismo, y en el caso particular del Proyecto Mirador, también a los desalojos de personas que habitan en las áreas de influencia directa del proyecto.

\section{Mujeres shuar, conflicto y extractivismo}

La industria extractivista no solo tiene un impacto económico, sino que incide directamente en la sociedad shuar acelerando su aculturación ${ }^{3}$ y haciendo desaparecer el modo de vida con el que este pueblo ha garantizado su supervivencia a lo largo del tiempo en la selva amazónica. Este impacto social se expresa también en la transformación de las relaciones de género. La transformación del sistema productivo y la contaminación de los recursos naturales son factores que pueden tener un impacto diferencial sobre mujeres y hombres cuando hablamos de contextos en los que el género es una variable social de notable importancia y en los que además se da una relación significativa entre las mujeres y los recursos naturales, pues son estas las encargadas de conseguir el alimento para el cuidado de la familia (Poats, 1999: 143). Desde la visión ecofeminista de Vandana Shiva y Maria Mies las mujeres rurales e indígenas han cumplido un papel muy importante como cuidadoras de la vida y del

El concepto de "aculturación" se refiere en este artículo a la pérdida de hábitos, prácticas y valores culturales en el marco de un sistema socio-económico particular por la adopción de otros, que en este caso implican diferentes formas de entender y manejar el territorio a partir de la monetarización de la economía indígena (Gudynas, 2009; Ortiz, 1997). 
medio ambiente, lo que a menudo ha conllevado su mayor necesidad de preservar los recursos naturales para proteger su propio bienestar. Este planteamiento asume en la experiencia femenina de los países del "Sur" una mayor cercanía con la naturaleza, y relaciona la expansión del capitalismo en estos contextos con el aumento del sexismo y la discriminación de género:

El género y la diversidad están vinculados en muchos aspectos. La construcción de las mujeres como el «segundo sexo» está asociada a la misma incapacidad para aceptar la diferencia que se encuentra en la base del paradigma del desarrollo que conduce al desplazamiento y la aniquilación de la diversidad en el mundo biológico. El mundo patriarcal considera al hombre como medida de todo valor y no admite la diversidad, sino la jerarquía. Trata a la mujer como desigual e inferior porque es diferente. No considera intrínsecamente valiosa la diversidad de la naturaleza en sí misma, sino que solo su explotación comercial en busca de un beneficio económico le confiere valor. [...] La marginación de las mujeres y la destrucción de la biodiversidad son procesos que van unidos (Mies y Shiva, 1998: 13).

El ecofeminismo revela de hecho la importancia de poner a las mujeres del "Sur" en el centro del discurso sobre el desarrollo, defendiendo la necesidad de un modelo sostenible tanto desde el punto de vista ecológico como del humano (León, 2008: 36). Y aunque particularmente el ecofeminismo de Shiva ha sido ampliamente debatido por resultar esencialista, también ha visibilizado el carácter patriarcal de la globalización capitalista, y ha puesto en evidencia el hecho de que el discurso científico se construya con categorías y paradigmas que no reflejan las preocupaciones, prioridades y percepciones femeninas (Shiva, 2006). Como respuesta a esta realidad, se señala la conveniencia de introducir en la ciencia moderna nuevos símbolos y valores que permitan la participación de las mujeres rurales e indígenas en la producción de conocimiento (Puleo, 2011; Gargallo, 2014; Cuvi, 2000).

Por otro lado, el hecho de que también las feministas indígenas cuestionen la forma de categorizar la realidad del discurso académico, rechazando incluso el concepto de igualdad occidental, no ha de llevarnos ingenuamente a pensar que el sistema de género que defienden sea el representado por las culturas precolombinas. Cuando hablamos de sociedades indígenas de América Latina, frecuentemente nos referimos a contextos donde existe un "patriarcado entroncado" o "mixto", utilizando los conceptos del feminismo comunitario (Gargallo, 2014; Cabnal, 2010) para aludir a un sistema que combina elementos que se heredan del colonialismo con elementos de patriarcados ancestrales. El feminismo comunitario rechaza la explotación del territorio ancestral de la misma forma que la violencia contra las mujeres (Gargallo, 2014; Paredes, 2008; Cabnal, 2010), en un lenguaje diferente al del feminismo clásico, tratando de hacer compatible la defensa de los derechos individuales de las mujeres con los derechos colectivos de los pueblos y nacionalidades (Cabnal, 2010).

Es decir, para analizar las respuestas de las mujeres shuar a las nuevas condiciones creadas por la extensión de las actividades extractivas llevadas a cabo por empresas transnacionales en su territorio, es necesario entender cómo se han expresado tradicionalmente las relaciones de género. La sociedad shuar ha tenido históricamente una forma especialmente individualista de entender el prestigio social, unida a la 
idea de fuerza o de poder (kakáram) (Mader, 1999: 99)4. La concepción del poder como atributo de la persona se ha reflejado tradicionalmente en un orden social no estratificado en el que el estatus social dependía básicamente de cualidades personales, conjuntamente con la edad y el género (Mader, 1999: 37). Entre estas cualidades, el carisma y la popularidad han sido altamente valoradas, por estar asociadas a la capacidad de influir sobre los otros, por lo que se consideran requisitos para ejercer posiciones políticas y de liderazgo (Mader, 1999: 348). También la intangibilidad e invulnerabilidad, así como la fuerza y superioridad, son vistas como cualidades que otorgan poder a la persona, aunque su expresión se asocia a contextos de actividad diferentes para cada género (1999: 348). Normalmente los hombres shuar adquirían prestigio siendo buenos cazadores, pescadores, o guerreros, y teniendo muchos hijos (Hendricks, 1996: 137; Mader, 1999: 28). Las mujeres alcanzaban buena fama mediante su habilidad para elaborar chicha o cuidar el huerto, así como por su procedencia de una familia de prestigio (Mader, 1999: 28-29). Por otro lado, aunque la complementariedad y el equilibrio son rasgos predominantes de la relación entre hombre y mujer en las esferas económica y ritual, Mader resalta asimismo el dominio masculino sobre la política de alianzas y conflictos (1999: 35). Esta cuestión es importante para entender el contexto actual, ya que en general la participación de las mujeres shuar en reuniones y procesos comunitarios sigue siendo menor que la de los hombres (Poats, 1999: 150; Garcés, 2006: 61, 70). Además, aun cuando existe una notable participación femenina dentro de los movimientos indígenas, es común que los intereses de las mujeres queden invisibilizados (Poats, 1999: 143; Méndez, 2009: 65-66), y que estas tiendan a estar infra-representadas en los mecanismos establecidos para la participación de las poblaciones indígenas en los conflictos socioambientales, como en los procesos de socialización y de consulta.

No obstante, también es cierto que dentro de las relaciones sociales de los shuar se están dando cambios sustanciales que afectan especialmente a la forma en que se concibe la familia. Un estudio sobre las relaciones de género entre los shuar del Valle de Upano revela que existe ya una tendencia a fundar los matrimonios en la elección de pareja (en lugar de ser concertados) (Garcés, 2006: 62), así como un mayor acceso por parte de las mujeres a espacios, recursos y bienes, educación y actividades de mercado (Garcés, 2006: 65). Al mismo tiempo, este estudio señala algunos cambios negativos percibidos por las mujeres, como el aumento de la violencia por parte de los maridos, la menor colaboración mutua entre hombres y mujeres o la creciente falta de diálogo dentro de las familias (2006: 67). Otro de los estudios realizados en la última década en el contexto de los pueblos indígenas amazónicos de Ecuador señala la compleja problemática que conlleva la influencia de patrones globales en relación al concepto y prácticas de la feminidad en la población quichua de Napo (Muratorio, 2000). En este estudio Muratorio constata que la mayor libertad sexual de las adolescentes, atraídas por las nuevas formas de vida asociadas a las ciudades, es percibida por las mujeres más mayores de modo negativo. Aludiendo a la cuestión cultural, este cambio fortalece los procesos de deslegitimación de los mecanismos tradicionales de control de la sexualidad femenina, pero fomenta asimismo, tal y como lo ven las mujeres en el estudio de Muratorio, nuevos peligros para las jóvenes, pues en un contexto en el que estas son cada vez más depen-

$4 \quad$ El ritual de reducción de cabezas (tsantsa), practicado antiguamente por los shuar con sus enemigos también forma parte del imaginario de este pueblo, algo que les hizo ganarse la fama de fieros guerreros (Karsten, 2000: 225-229). 
dientes de los salarios de sus maridos, la prostitución aparece como un nuevo ámbito de la feminidad, asociada ahora a un nuevo y reduccionista concepto estético ${ }^{5}$.

En resumen, las respuestas de las mujeres shuar al conflicto socioambiental van a estar condicionadas por una serie de factores que también tienen que ver con la forma en que se ha entendido el género en las relaciones sociales tradicionales de la cultura shuar. Como indica Carmen Tene (2000), aspectos como la tradicional división sexual del trabajo, el limitado control de las mujeres sobre su sexualidad, o la forma en que cada pueblo concibe la participación femenina en el ámbito público, influyen de forma directa en el hecho de que las mujeres indígenas de Ecuador se hayan convertido en asalariadas muy pobres. En el caso de las mujeres shuar, estos aspectos exponen a las mujeres a unas condiciones particulares que a continuación analizo.

\section{Metodología}

Los datos que se manejan en este artículo se han producido en el marco del sub-proyecto Ecuador (focalizado en el Proyecto Mirador) de la investigación "Extracting Justice", coordinado desde el Observatorio de Conflictos Socioambientales (OBSA) de la Universidad Técnica Particular de Loja (UTPL) conjuntamente con la Norwegian University of Life Sciences ${ }^{6}$. "Extracting Justice. Explorando el papel de la consulta y el consentimiento previo, libre e informado (CsPLI), y la compensación, relacionados con los conflictos socioambientales en América Latina" es un proyecto de investigación internacional financiado por el Consejo Noruego de Investigación, que analiza los procesos de "consulta previa, libre e informada" en diversos contextos de extracción de recursos naturales situados en Bolivia, Colombia, Ecuador y Perú. En la UTPL el proyecto se inicia en el año 2014, si bien el OBSA trabaja desde el 2008 en la zona, año en el que se crea como respuesta a la creciente conflictividad socioambiental en el Sur del Ecuador debida en gran parte a la existencia de proyectos de extracción metalúrgica.

Particularmente en el caso del Proyecto Mirador en Ecuador, la investigación hace hincapié en cuatro aspectos fundamentales: 1) la existencia de mecanismos redistributivos y de compensación económica, 2) cómo estas prácticas redistributivas afectan a las actividades económicas de las comunidades indígenas y a su modo de vida, 3) cómo afectan a las políticas conservacionistas y del Buen Vivir, y 4) de qué manera aumentan o reducen el conflicto en torno a la minería a gran escala.

El análisis que aquí se expone está centrado en el cuarto aspecto mencionado. En este texto se analizan cuáles son los impactos que puede tener la minería a gran escala específicamente sobre las mujeres de la etnia shuar, población indígena afectada por el Proyecto Mirador, y cuál es su percepción en torno al conflicto que plantea. El interés por el conflicto desde una perspectiva de género ayuda a su vez a profundizar en las respuestas comunitarias dadas en este contexto, eje fundamental del proyecto, y parte a su vez del reconocimiento del factor cultural/étnico como aspecto que afecta transversalmente a cualquier hecho social, siendo de máxima importancia cuando hacemos referencia a grupos que han sufrido históricamente una gran discriminación.

5 Las mujeres indígenas de la Amazonía ecuatoriana se consideran asimismo población especialmente expuesta a la propagación del VIH por la falta de protección y control de su propia sexualidad dentro del matrimonio. Véase el artículo de Desirée Yépez "El VIH se expande entre las mujeres indígenas", publicado en la revista digital Plan $V$ el 15 de septiembre de 2015.

6 Institución a la que pertenece Esben Leifsen, director del sub-proyecto Ecuador. 


\section{Participantes}

Para este análisis se realizaron 21 entrevistas en profundidad entre 2015 y 2016, 20 de ellas a mujeres shuar que habitan en el área de influencia directa del Proyecto Mirador según el último informe facilitado por el Ministerio del Ambiente (Cardno, 2014), y una a la mujer (auto-identificada como mestiza) que presidió de 2006 a 2012 el Frente de Mujeres en Defensa de la Vida de El Pangui (cantón donde se sitúa el Proyecto Mirador) en la etapa de mayor activismo anti-minero en la zona.

Se resume aquí de forma algo más detallada la información sobre las 20 mujeres shuar entrevistadas:

- 4 mujeres de la comunidad shuar Etsa, perteneciente a la parroquia de Tundayme (cantón El Pangui)7. Según el "Borrador del Diagnóstico del Plan de Desarrollo y Ordenamiento Territorial de la Parroquia Tundayme, cantón El Pangui" (actualmente en elaboración) Etsa posee una población de 24 habitantes.

- 7 mujeres de la comunidad shuar Yanua Kim, también perteneciente a Tundayme, con una población aproximada de entre 30 y 40 habitantes, según el mismo borrador del DPOT de Tundayme.

- 7 mujeres de la comunidad Numpaim San Carlos, también en Tundayme y con una población similar a Yanua Kim.

- 1 mujer shuar habitante de la cabecera parroquial de Tundayme que pertenece a la organización CASCOMI (Comunidad Amazónica de Acción Social Cordillera del Cóndor). Esta organización se legaliza en 2014, coincidiendo con el derrocamiento del barrio San Marcos por parte de ECSA, empresa que detenta el proyecto de minería a gran escala en la zona, y en la actualidad es la que ejerce una mayor resistencia contra el Proyecto Mirador.

- 1 mujer shuar habitante de una de las comunidades vecinas que pertenece a la asociación Shuar El Pangui, dentro de la cual es representante de las mujeres shuar del cantón. Esta asociación se declara neutral ante el conflicto socioambiental en la zona, así como mediadora entre la población shuar afectada y enfrentada por su posicionamiento a favor o en contra del extractivismo. Sin embargo, gran parte de la población shuar de las comunidades donde se realizaron las entrevistas acusa a esta asociación de haber servido a los intereses de la empresa.

\section{El papel de las mujeres shuar dentro del conflicto socioambiental asociado al Proyecto Mirador}

\subsection{Importancia del sistema de valores shuar en el discurso femenino en relación al conflicto}

Cuando analizamos el impacto social que producen las actividades extractivas sobre las poblaciones indígenas damos relevancia al hecho de que estas poblaciones, no solo se enfrentan a una profunda transformación de su economía por la invasión de

\footnotetext{
7 De acuerdo al "Borrador del Diagnóstico del Plan de Desarrollo y Ordenamiento Territorial de la Parroquia Tundayme, cantón El Pangui” (en elaboración por parte del GADM de Tundayme), esta parroquia cuenta con 854 habitantes censados, de los cuales $163(22,12 \%)$ se auto-identifican como indígenas.
} 
un modelo productivo ajeno, sino que además este modelo impone unos valores radicalmente diferentes a los que han formado parte históricamente de su cultura. Sin embargo, es fácil pasar por alto esta cuestión cuando se aplica el paradigma de desarrollo occidental, sin tener en cuenta los valores intrínsecos de la cultura shuar, o de aquellos asociados particularmente a la experiencia de las mujeres shuar. Este aspecto es tratado en profundidad por Gargallo (2014: 18) cuando critica el tratamiento académico de las mujeres indígenas, aludiendo a la necesidad de abarcar la complejidad que plantea la existencia de una base conceptual no compartida. Es decir, en la amplia diversidad de pueblos indígenas de América Latina, las culturas no presentan de forma general una supremacía de lo humano sobre lo natural de la misma manera que la cultura occidental, ni tampoco una lógica utilitarista ni individualista. Tampoco reconocen como valores propios o positivos en la mayoría de los casos la igualdad entre hombre y mujer, sino la complementariedad, ni el ideal de transcendencia a través del trabajo profesional (Gargallo, 2014; Del Campo, 2012). El choque que produce la coexistencia de dos modos de vida marca considerablemente las respuestas dadas al conflicto socioambiental en el contexto shuar. Específicamente, al escuchar el discurso de las mujeres shuar llama la atención la presencia de tres elementos que se contraponen a los valores presentes en el discurso occidental sobre desarrollo: concepto del bienestar desligado de la ética individualista y del trabajo desde el punto de vista occidental, ideal de bienestar de la naturaleza, y reivindicación de la importancia del papel femenino (frente al ideal de igualdad de género o frente a la crítica de la división sexual del trabajo, algo propio del discurso occidental).

\subsubsection{Concepto del bienestar desligado de la ética individualista y del trabajo desde el punto de vista occidental}

Uno de los cambios más importantes de los que las mujeres shuar nos hablan tiene que ver con la mayor dependencia de las comunidades del trabajo asalariado. Hay que tener en cuenta que en los contextos donde las mujeres tienen una mayor dificultad que los hombres para acceder a puestos de trabajo remunerado, es más probable que sean ellas las que perciban la influencia de la minería en las actividades cotidianas de la comunidad como algo negativo y que sufran una mayor pobreza. Pero, aun cuando las mujeres pueden acceder como los hombres a un puesto de trabajo en las transnacionales, este les obliga a adoptar unas normas y valores que generalmente no comparten y que tienden a romper las dinámicas colaborativas anteriores. Una mujer shuar de 48 años señala que antes las mujeres estaban más unidas; a menudo "los hombres hacían mingas y todas las mujeres se reunían y colaboraban". Por el contrario, "ahora las familias son más individualistas", no hay apenas actividades en común y es difícil ponerse de acuerdo en temas que atañen a la comunidad. En general, el posicionamiento de las mujeres a favor o en contra de la minería depende de la dependencia económica que tengan de esta, ya que muchas de ellas trabajan para la empresa minera, principalmente en la cocina, limpieza o lavandería ${ }^{8}$, aunque también es común encontrar entre estas mujeres una postura crítica hacia el extractivismo.

\footnotetext{
Este aspecto no afecta de la misma manera a la comunidad de Numpaim San Carlos, que se encuentra algo más lejos de la empresa que las otras dos comunidades y tiene mayores dificultades de acceso. Las mujeres de este centro aseguran que en su comunidad nunca nadie ha querido trabajar en la minería a gran escala, lo que hace que su posicionamiento en contra de ella sea mucho más homogéneo.
} 
Como explica la ex presidenta del Frente de Mujeres en Defensa de la Vida de El Pangui, las transnacionales traen consigo un modo de vida que no respeta la naturaleza y que los convierte en pobres, al despojarlos de los recursos naturales de los que depende una economía agrícola y ganadera.

Nosotros quedaremos más pobres que antes porque nosotros hemos vivido de la ganadería, la agricultura. Viene la contaminación, porque a cielo abierto se contamina todo, entonces todos nuestros cultivos se perderán, entonces de qué vamos a vivir, tendremos que emigrar a otra parte. (...) Las transnacionales qué hacen, vienen, se llevan todo, nos dejan pobreza, contaminación y muerte, porque nosotros con el tiempo tendremos que salir de El Pangui (Ex presidenta del Frente de Mujeres en Defensa de la Vida de El Pangui).

Las mujeres entrevistadas que viven todavía en sus comunidades de los productos de la tierra no comparten el ideal de forma de vida basado en la ética individualista y del trabajo desde el punto de vista occidental. La mayoría de ellas no desea alejarse de sus territorios reconocidos como ancestrales, ni abandonar la vida rural. Salir de su lugar de su origen es percibido por estas mujeres como la verdadera amenaza de pobreza, en contraste con la visión que entiende su inserción en el mercado laboral como progreso y desarrollo.

\subsubsection{Ideal de bienestar de la naturaleza}

Los trabajos antropológicos han tendido a representar las culturas amazónicas como cuidadoras legítimas de la naturaleza por la forma en que han mantenido durante milenios un modo de vida en equilibrio con el medio natural (Villena, 2000). Esta visión debe ser ampliada cuando hacemos referencia a grupos concretos que, como los shuar de Zamora Chinchipe, han practicado tradicionalmente actividades productivas que dañan la naturaleza: los shuar han explotado la minería artesanal o la tala de madera, actividades prohibidas o sometidas a una mayor regulación en la actualidad, y han apoyado la introducción de ganado vacuno en el ecosistema amazónico, lo que ha tenido a su vez un impacto ambiental negativo.

Pero, a pesar de las contradicciones que plantea el discurso etno-ecologista aplicado a la situación de las comunidades shuar, lo cierto es que en las últimas décadas ha sido muy frecuente la cooperación entre colectivos shuar/campesinos y ecologistas, y particularmente las mujeres, aunque hayan participado en menor medida que los hombres en acciones colectivas que implican cierta movilización y contactos fuera de la comunidad, utilizan por lo general un discurso que apoyaría este ideal de bienestar de la naturaleza. La defensa de la naturaleza, en el caso que tratamos, ocurre principalmente en función de las necesidades humanas, cuando la destrucción y la contaminación afectan directamente a la supervivencia de las comunidades, de la forma que expresa el concepto de "ecologismo de los pobres" (Martínez-Alíer, 2004).

$9 \quad$ El "ecologismo de los pobres" es un concepto útil cuando se estudian sociedades en las que el modo de vida implica una interdependencia material y simbólica de seres humanos y humanos y naturaleza, lo que se expresa en prácticas ecológicamente sostenibles. No obstante, aquí no se pretende medir el nivel de ecologismo de los shuar, sino entender la importancia que ha tenido y todavía sigue teniendo el vínculo con la naturaleza en las actividades, hábitos y modos de sentir asociados a su economía, conocimiento y espiritualidad (Guerrero, 2011: 93). 
Yo mantengo a mi gente, a mi familia, con la ganadería y la agricultura, tengo 65 años y tengo que seguir trabajando y si toca decir no a la minería a gran escala pues lo digo. Y les he pedido yo a mis compañeros y a la generación que está viniendo que tomen conciencia, que piensen, que lo que nos están haciendo no está bien, porque donde se está haciendo la minería a gran escala era el Parque Binacional de la Paz, allí hay plantas que en ninguna parte del mundo hay, plantas medicinales, plantas ornamentales, aves que en ninguna parte del mundo hay, hay las ruinas arqueológicas que no debían ser tocadas pero nadie ha hecho nada (Ex presidenta del Frente de Mujeres en Defensa de la Vida de El Pangui).

En este sentido, es innegable el protagonismo de las mujeres indígenas en la defensa del medio ambiente, del que dependen para poder realizar los trabajos de alimentación y cuidado de la familia, o la venta de productos de su huerta en los mercados. Una mujer de 24 años de Yanua Kim nos explica que la productividad de sus tierras ha disminuido además de forma notable a causa de una inundación que ella relaciona con la construcción de una presa por parte de la empresa minera, y que afectó a las huertas de esta comunidad: "El río está sucísimo, a mi huerta le ha afectado bastantísimo, las plantas se habían muerto, me tocaba resembrar y así está, ahora tengo menos productos, más antes sí vendía, ahora ya no vendo en el mercado, se inundó el río todito y por eso ya no vendo".

También es común que las mujeres shuar expresen un ideal de bienestar basado en el equilibrio con la naturaleza, en oposición al sistema de acumulación de bienes y prestigio que perciben como destructor de la vida. En el siguiente testimonio, una mujer shuar joven nos traslada este ideal de felicidad que ella vincula a la posibilidad de acceder a los recursos de la naturaleza.

Yo prefiero tener poca plata y vivir en un ambiente sano para mis propios hijos. La gente blanca, ellos solo quieren dinero, dinero y dinero, supuestamente con eso va a haber el progreso. (...) Me gustaría que toda la gente pensara que no es bueno dañar la naturaleza, no entiendo, con ver que hay plata tienen que destruir y destruir hasta que todo lo dañan. Yo digo a veces también que nuestro Señor se decepciona de nosotros porque somos tan ambiciosos, pudiendo cuidar y disfrutar de lo mejor, de lo que hay aquí en este mundo que Dios nos ha dado, lo destruimos. Yo estoy sin plata, pero yo me siento feliz, estoy contenta porque tengo un plátano sano, una yuca, un pollo, tengo para comer, tengo cultivado y sé que voy a comer. Tengo la naturaleza completa (mujer shuar, 25 años, Yanua).

El hecho de dar a la naturaleza un valor meramente económico es interpretado por esta mujer de forma negativa y equivocada. En su discurso coloca la naturaleza en un plano superior con respecto a los seres humanos que, en su opinión, lejos de aspirar a su dominio para conseguir desarrollo, han de aprender a colocarse humildemente en un plano de inferioridad, de acuerdo a su dependencia. La misma mujer afirma: "Nos dicen jíbaros, con eso quieren decir que nosotros somos salvajes, no saben de desarrollo, no es así, a veces los jíbaros son más conscientes que los estudiados, ¿por qué?, porque nosotros los jíbaros aprendemos de nuestra tierra a ser humildes". 


\subsubsection{Reivindicación de la importancia del papel femenino}

A pesar de que los roles de género están siendo transformados por las influencias diversas que afectan a las comunidades indígenas shuar, y de que en esta transformación está presente la crítica al machismo asociado con las tradiciones antiguas, la forma de entender este cambio no puede equipararse al discurso occidental construido sobre la crítica radical a las identidades de género basadas en la oposición de los sexos.

Los choques experimentados a partir del contacto del mundo shuar con las dinámicas modernas producen, por un lado, la minimización de las diferencias percibidas entre las características de lo "masculino" y lo "femenino". Y, por otro lado, dado que esas características son asociadas a su vez a la propia cultura, no es frecuente que entre las mujeres indígenas se manifieste un auténtico deseo de superar las funciones históricamente asignadas en su pueblo a lo femenino, tal y como se ha dado en la cultura occidental. Esto significa que, aunque ciertos cambios sean tolerados sin dificultad, como el hecho de que hombres y mujeres quieran acceder a un empleo remunerado, o participen indistintamente en actividades que antes formaban parte de los roles de género, sigue existiendo en las mujeres el orgullo de poder realizar el duro trabajo que requiere el campo y la maternidad. Por ejemplo, una mujer de 35 años de Numpain San Carlos nos dice: "tengo mis niños en la escuela, paso lavando la ropa, para sembrar yuca, por ejemplo, se tumban los árboles, de ahí se saca la leña, eso lo hacen las mujeres, tienen que hacer todo lo posible para cocinar. El agua la pusimos nosotras en todas las casas, es agua de la quebrada". A partir de esa condición, también es común que se idealice la protección por parte del varón, como algo merecido.

\subsection{Cambios en las relaciones de género}

Con relación a los cambios en las relaciones de género, en las entrevistas realizadas se ha hablado de cuestiones que ponían en evidencia dos aspectos importantes: los cambios producidos en la familia, la afectividad y la sexualidad, y la existencia de una gran presión social como principal mecanismo de control sobre las mujeres shuar. Ambos aspectos marcan al mismo tiempo la forma en que se interpretan los conflictos sociales asociados a las nuevas formas de vida y comportamiento que genera la industria extractivista en las comunidades.

\subsubsection{Cambios en la familia, la afectividad y la sexualidad}

El pueblo shuar era tradicionalmente polígamo (en particular hablamos de poliginia). Esta estructura familiar, sin embargo, ha dejado de ser significativa en los shuar que habitan en Ecuador, debido en gran parte a la influencia que recibieron de los salesianos en el proceso de creación de los centros shuar (Rubenstein, 2005) ${ }^{10}$.

En la actualidad, no se registra ningún matrimonio polígamo en las comunidades visitadas (se hace referencia únicamente a casos de personas ya fallecidas). Tenemos

10 Antes de la creación de los centros, los shuar habitaban dispersos practicando la caza y la recolección. Esta organización empezó a cambiar cuando los ecuatorianos de la sierra empezaron a asentarse en la Amazonía a finales del siglo XIX (Rubenstein, 2005: 29). 
conocimiento de algunos matrimonios religiosos ${ }^{11}$, pero la tendencia más importante es la práctica que llaman "unión libre": un hombre y una mujer comienzan a vivir juntos sin realizar ningún trámite administrativo previo. La preferencia de los shuar por la unión libre favorece el retraso de las uniones conyugales, pues hasta hace poco tiempo la costumbre era casar a las niñas con 13-14 años con hombres de mayor edad. Al mismo tiempo, permite que sean más frecuentes las separaciones, que aunque continúan siendo mal vistas, ya se interpretan como inevitables entre las jóvenes, quienes a menudo expresan su malestar por la afición de los hombres shuar a buscar mujeres fuera del matrimonio o unión.

En general, se puede decir que las mujeres shuar rechazan de forma generalizada el matrimonio tradicional, tanto por el hecho de que las mujeres tuvieran que compartir marido como por que con frecuencia eran casadas a la fuerza siendo todavía niñas. En las líneas que siguen una mujer shuar relata su experiencia. Esta mujer, de 31 años, escapó de un matrimonio forzoso con un hombre treintaicinco años mayor que ella con el que tuvo cinco hijos. "Yo me casé de 13 años. Yo era inocente cuando me casé con mi marido, o sea, mi mami, mi papi, ellos me obligaron a casar, yo me casé a la fuerza, por obligación". Su actual compañero explica cómo el hombre podía elegir una niña para casarse, comprometiéndose a alimentarla con el producto de su cacería hasta que la niña crecía. Ella continúa: "Él tenía como 48 años. Todos esos años lo tuve que aguantar. No me trataba bien, me maltrataba, bueno, pegar no, pero maltrato psicológico sí. Trabajar sí me dejaba, pero salir, dar un paseo, no."

Las mujeres shuar miran el pasado de su pueblo con alivio por percibir que se encuentran en una mejor situación. Por otro lado, también es cierto que es raro que una mujer shuar pueda ejercer control sobre su propia sexualidad. Por ejemplo, una joven embarazada de su tercer hijo reconoce que le gustaría no tener más, pero a la vez plantea la dificultad de conseguirlo debido al descuido de su pareja. Esta mujer se posiciona totalmente en contra de la práctica de la poligamia:

En primer lugar, yo digo por mí misma, a mí no me gusta compartir a mi marido con nadie, está bien conmigo o no está conmigo, si está con alguien que coja y se vaya, (...) las mujeres antiguas toleraban tanto machismo al marido, cómo hacer si, supongamos que él tenía 4 mujeres, un día dormía con ella, el siguiente día con ella, al otro día con ella y al cuarto día con ella. En ese caso, en estas horas de la tarde, la de allá, de allá, de allá y de acá, le venían a servir la chicha, a comer entre todas, si tienen hijos a servirse todos juntos, y ahora yo me pregunto cómo ellas no tenían asco (mujer shuar, 25 años, Yanua Kim).

El rechazo a las costumbres shuar que las mujeres consideran machistas las lleva cada vez más a menudo a establecer relaciones sentimentales con hombres de fuera de su entorno, circunstancia que también puede acompañar la búsqueda de empleo en trabajos remunerados. Esta cuestión se relaciona con dos fenómenos percibidos entre las mujeres entrevistadas: 1) el hecho de que el trabajo femenino en la empresa puede fomentar el abandono familiar, puesto que la mujer dispone de dinero propio, y 2) la percepción de una mayor discriminación y maltrato a las mujeres shuar fuera

11 La mayoría de las personas de estas comunidades se declara de religión católica o evangélica. 
de la comunidad. Esta experiencia es aludida, de hecho, como motivo de vuelta a sus comunidades por varias mujeres que pasaron años trabajando fuera de Tundayme conviviendo con hombres mestizos.

Asimismo podemos relacionar las ventajas de la unión libre, no solo con la posibilidad para las mujeres de poner fin a una relación insatisfactoria (algo que no ocurre sin poner en riesgo la reputación de la mujer), sino también con la creciente dependencia económica que tienen las mujeres de los hombres en las zonas donde estos se han incorporado en mayor medida en los trabajos disponibles desde el asentamiento de la empresa minera en la zona. Particularmente estas zonas son también las más afectadas por los cambios percibidos por las mujeres en tierra, agua y fauna que limitan su capacidad productiva mediante el uso de sus propios recursos.

\subsubsection{La presión social (o reputación) como principal mecanismo de control sobre las mujeres en las comunidades shuar}

Y, si bien es cierto que las nuevas condiciones económicas pueden llegar a transformar las relaciones de género, también es necesario admitir que en la vida cotidiana de las comunidades shuar se ejerce todavía una considerable presión social sobre las mujeres. En la cultura shuar la vida familiar ha garantizado siempre una esfera femenina que permitía cierta autonomía a las mujeres (Karsten, 2000: 199), pero al mismo tiempo el incumplimiento del rol femenino ha sido cruelmente castigado. Por ejemplo, la mujer adúltera podía ser castigada hasta la muerte si su marido disponía ejercer así su derecho a la venganza sobre ella (Karsten, 2000: 177). En la actualidad, de todos los aspectos que intervienen en la reputación de la mujer, este es quizá el que más censura acarrea.

Especialmente en las áreas más cercanas a la empresa, donde las personas tienen una mayor relación con el mundo mestizo y hay cada vez más mujeres que han vivido o trabajado fuera de la comunidad, las mujeres que cometen infidelidad o las que abandonan el matrimonio, se enfrentan a la marginación social ${ }^{12}$. Estas rupturas están motivadas en muchas ocasiones por el sufrimiento de maltrato de sus maridos, aunque este constituye un tema tabú del que la mayoría de mujeres de las comunidades shuar prefiere no hablar ni relacionar con los abandonos familiares. Incluso las mujeres que sí llegan a relatar actos de violencia de su pareja contra ellas, pueden llegar a rechazar la decisión de otras mujeres de terminar con esta clase de situaciones $^{13}$. Muchas mujeres jóvenes, sin embargo, sí relacionan la violencia doméstica con las separaciones cuando manifiestan su preferencia por la unión libre, y no el matrimonio, por ser más fácil de romper. Estas mujeres asocian a su vez la violencia que ejercen los hombres en el espacio doméstico con el consumo de alcohol.

Y, si bien la intimidad de las mujeres aparece como el aspecto más sometido a control social a primera vista, también se ha de considerar que esta misma presión social se puede estar expresando en la escasa visibilidad de portavoces femeninas shuar en un escenario tan directamente afectado por la actividad extractivista, y donde la resistencia anti-minera por parte de los shuar ha sido notable.

12 En relación con esta cuestión, se puede señalar la dificultad que tenemos como investigadores/as para acceder a la conversación con estas mujeres, pues aunque hayan vuelto a vivir a la comunidad, no participan como el resto en actividades colectivas ni acceden a hablar de sus vidas.

13 "Yo no soy de la clase de mujer que abandonaría el hogar y no me he casado, pero una mujer tiene que dar ejemplo y sacrificarse. Ahora hay muchas mujeres que se abandonan a sus maridos" (mujer shuar, 38 años, Yanua Kim). 


\subsection{Pérdida de la responsabilidad femenina sobre la reproducción cultural en el proceso de aculturación}

A menudo se hace referencia al papel activo que tienen las mujeres como reproductoras de la cultura en las sociedades indígenas de América Latina. Particularmente en la cultura shuar se puede decir que este papel ha sido importante. Del papel femenino podemos resaltar su función complementaria con respecto a lo masculino en las esferas económica y ritual (Mader, 1999: 35), así como la importancia social de las actividades que tradicionalmente eran propias de las mujeres, especialmente de la elaboración de la chicha y del cuidado del huerto. De estas actividades dependía además el prestigio otorgado a la mujer shuar (Mader, 1999: 28-29). Karsten (2000) destaca la autonomía de la que las mujeres disfrutaban por el hecho de tener un acceso directo a los productos de la huerta. Y otros trabajos sobre las culturas amazónicas han señalado la importancia que el rol femenino tenía en el mantenimiento de unas relaciones armónicas con la naturaleza y el mundo espiritual. Por ejemplo, la obligación de reciprocidad con la naturaleza era practicada por las mujeres en el acto de dar a luz en la huerta (Descola, 1996: 294); las mujeres también eran las encargadas de criar como si fueran "hijos" a los perros (empleados en la caza) y animales de la selva que se adoptaban como mascotas (Karsten, 2000: 141; Descola, 1996: 315-319). Por otro lado, la asimilación de la feminidad con la propia tierra, por el acto de la creación, otorgaba a las mujeres un especial protagonismo en el mundo simbólico, repleto de deidades femeninas con responsabilidad sobre casi todos los actos de la vida de los shuar (Pellizzaro et al., 2009).

Todos estos significados comienzan a cambiar de un modo significativo a mediados del siglo XX, en un proceso de aculturación masiva de los pueblos indígenas amazónicos en Ecuador (Costales y Costales, 2006: 141; Barriga, 1986: 34), que resulta particularmente grave en la provincia de Zamora Chinchipe por el intenso desarrollo de la actividad extractiva. Hablamos de un momento en el que a su vez se extienden los valores mercantiles y las culturas indígenas acusan una gran discriminación que impulsa finalmente la lucha de los movimientos indígenas en el país, que reclaman reconocimiento y autoafirmación. La aculturación en los shuar aumenta sus dificultades para responder conjuntamente a los problemas a los que se enfrentan como sociedad, provocando además una pérdida de autoestima y la aceptación del tipo de desarrollo traído por las transnacionales como modelo ideal.

En el caso de las mujeres, la pérdida de referentes culturales puede incluso afectar más directamente por los cambios que atraviesa la familia, como veíamos anteriormente. Por ejemplo, aunque en la actualidad las chicas tienen la posibilidad de casarse con el hombre que elijan, y a una mayor edad, entre las adolescentes solteras aumentan los casos de embarazos. Las niñas también pueden educarse en la escuela como los varones pero su motivación va a estar condicionada por diversos factores. Además, el aprendizaje de las habilidades que antes otorgaban estatus a las mujeres en la cultura shuar ya no es valorado, por lo que una mujer finalmente puede acabar teniendo hijos de diferentes hombres sin desarrollar un papel ni valorado ni propio de la cultura shuar tradicional. El testimonio que sigue es de una mujer de 19 años con 3 hijos, unida con un hombre que trabaja temporalmente en las obras que genera la empresa minera.

Aquí, le ayudo a mi mamá, cualquier cosita ya está limpiando, cuando se vive en el pueblo no haces ni eso. Nunca me dio ganas de estudiar, salí de la escuela, acá pasaba con mis papás ayudándoles un poco, y tuve a mi hija, madre soltera, así 
estando un año y seis meses conocí a mi marido. (...) Pero hay hombres que por más se casen están con otras, y yo le digo a él: si estás con otras mejor ándate, vive solterito, yo no te hice que te cases conmigo a la fuerza, pero ahora si estás conmigo tienes dos hijos, tienes que trabajar y asentar cabeza porque yo no como tierra, le digo, yo sé comer, mijito, mi papá me ha mantenido, cuando yo era soltera a mí nunca me faltó un grano de arroz en la casa, es más, por mí y por tus hijos, trabajando se tiene, si está echado cómo va a sacar siquiera un dólar. Y él eso no pensaba antes, él solo en el juego, en sus amistades, así le dije, si no quieres trabajar nos separamos, y así ya sentó cabeza, mi papá también le ha conseguido trabajo (mujer shuar, 19 años, Etsa).

Este discurso refleja el aumento de la dependencia económica de las mujeres con respecto a los varones en las zonas más cercanas a la empresa minera. Recordemos que es más frecuente el empleo de los hombres en esta industria, y que en la medida en que esta avanza, las mujeres perciben más dificultades para trabajar la tierra tal y como lo hacían antes. Las mayores dificultades de acceso a las nuevas fuentes de empleo para las mujeres produce lo que el ecofeminismo percibe como una feminización de la subsistencia (Mies y Shiva, 1997: 35) en un proceso en el que se devalúa simultáneamente lo femenino y la naturaleza, y en el que la ruptura del sistema económico anterior impide que las actividades de subsistencia que realizan las mujeres puedan garantizar lo que consideran una vida digna. En particular, las condiciones que afectan al modo en que se desarrolla el Proyecto Mirador han tenido como efecto una "re-patriarcalización" de los territorios afectados, tal y como señala el Colectivo de Investigación y Acción Psicosocial Ecuador (2017). Este estudio realizado en el área más cercana a la minera constata algunos hechos que potenciarían este reforzamiento del dominio masculino a través del extractivismo, como el aumento de la inseguridad femenina en áreas notablemente masculinizadas, o la exclusión de las mujeres tanto en el nuevo mercado laboral, como en las negociaciones y procesos de interlocución desde los cuales se toman las decisiones políticas. Este trabajo destaca asimismo la implantación de un prostíbulo en el área minera, aspecto que también es objeto de preocupación para las mujeres shuar, quienes lo asocian al aumento de enfermedades venéreas y a la infidelidad.

Por otro lado, la mayoría de las mujeres rechaza trabajar en la empresa por las duras condiciones que impone.

Sí estuve trabajando en la empresa, no le puedo mentir, 4 años estuve trabajando, después ya salí, ya no me gustó, yo estuve trabajando en la cocina, después estuve de obrera, y ya salí, es que a mí en la cocina me tocaba de noche, me iba de aquí 3 de la mañana a hacer el desayuno a los trabajadores, yo les dejaba aquí a mis chiquitos, 3 de la mañana ya sabía estar yéndome de aquí en la camioneta. Después, dentro de un año ya habían cambiado a mis compañeros en otro horario, sí, así pasé, por eso dejé de trabajar (mujer shuar, 24 años, Yanua Kim).

Otra de las mujeres shuar entrevistadas (de 38 años y embarazada del hombre con el que convive durante 23 años y con el que tiene 11 hijos) relata un episodio de mucha dureza relacionado con las malas condiciones de trabajo en la minería. Esta mujer trabajó durante algunos años junto a su marido en la empresa minera, hasta 
donde llevaba a una de sus hijas pequeñas. Cuenta que entonces la empresa habilitaba una carpa con colchones para la estancia de los niños de las madres trabajadoras y que un día, al salir del trabajo, encontró a su niña calcinada en este lugar tras haberse producido un accidente ${ }^{14}$.

En definitiva, en un contexto cultural donde tradicionalmente se asignaba a las mujeres el cuidado de las personas, la tierra y los animales, excluyéndolas de los mecanismos políticos de toma de decisiones, pero otorgándoles un estatus especial dentro de un sistema que buscaba la armonía con la naturaleza, el conflicto socioambiental tiende a reforzar la vulnerabilidad social de la población femenina, en parte por la pérdida de estatus de su rol tradicional. Esto no significa que las mujeres indígenas anhelen un estado ideal anterior; más bien nos ayuda a entender que su deseo por que les sea reconocida la importancia de su trabajo en la vida de las comunidades, obedece a la percepción de su devaluación y a la transformación de un sistema cultural en el que la reproducción de la vida y la cultura ya no recae exclusivamente sobre la familia, teniendo como centro lo femenino ${ }^{15}$.

\subsection{Las mujeres shuar ante un futuro amenazado por la minería a gran escala}

La empresa minera EcuaCorriente (ECSA) está asentada en la Cordillera del Cóndor desde 1999, año en el que se inician los primeros trabajos de exploración avanzada y también los primeros conflictos (Colectivo de Investigación y Acción Psicosocial Ecuador, 2017: 46) ${ }^{16}$. A partir de entonces todas las actividades llevadas a cabo se han desarrollado sin ningún proceso de consulta, lo que provocó la organización de los habitantes de la zona, tanto mestizos como shuar, en grupos de defensa de su territorio, apoyados frecuentemente por grupos ecologistas. Las mujeres en esta región, especialmente las mujeres shuar, participaron activamente en las movilizaciones contra la minería a gran escala reclamando respeto a sus tierras y cultura. Estas reivindicaciones fueron duramente reprimidas por el Estado, pero a su vez permitieron escuchar las voces de aquellas personas que sufren más directamente las consecuencias de las actividades extractivas en la Amazonía, en sociedades en las que no era común la participación política de las mujeres.

En 2006 se constituye el Comité en Defensa de la Vida, la Salud y la Naturaleza, así como el Frente de Mujeres en Defensa de la Vida de El Pangui, que se mantienen bastante activos hasta el año 2012. Esta última organización llegó a agrupar alrededor de 20 mujeres del cantón El Pangui, mestizas y shuar. Al hablar específicamente de la posición de las mujeres con respecto a la minería, o sobre las acciones en las que han participado a lo largo de los años en los que está presente el conflicto, la ex presidenta del Frente de Mujeres en Defensa de la Vida de El Pangui destaca la especial fuerza de las mujeres shuar y su liderazgo en los movimientos de reivindicación de sus tierras ancestrales. También explica lo que supuso la resistencia colectiva para una población diversa con poco grado de unión.

14 Esta misma mujer también perdió a otra hija en un accidente fuera de la empresa. La niña, que nació sin un brazo, murió ahogada en una pecera a los 10 años. La madre expresa que en parte fue un alivio pues donde vive son muy comunes los abusos sexuales sobre las niñas discapacitadas.

15 Se ha de tener en cuenta que las poblaciones shuar no viven en un aislamiento geográfico total, sino que sus viviendas se encuentran cada vez más cercanas a las vías y a los centros educativos, donde envían a los niños/ as a estudiar.

16 ECSA, empresa originalmente canadiense, fue absorbida por dos empresas chinas a finales de 2009. 
En El Pangui solo nos hemos unido a través de este problema que hubo, porque como nosotros hemos sido agricultores, ganaderos, hemos vivido de eso, entonces vino y nos juntamos y dijimos: vamos a defender lo que nuestra madre tierra nos ha dado, y formamos el Comité. Las mujeres reclamábamos que no haya la minería a gran escala, no queremos proyectos de minería a gran escala. Nosotros hemos visto que la minería artesanal podía ser porque en verdad es para nuestros compatriotas, a pesar que nosotras tampoco hemos estado de acuerdo pero en último caso hemos visto que por lo menos trabajan artesanalmente y ganan para nosotros también (...). Yo, de mi parte, estoy consciente y seguiré consciente de que nosotros lo que necesitamos es que nos dejen libres, que nos dejen trabajar nuestra madre tierra porque de eso vivimos (Ex presidenta del Frente de Mujeres en Defensa de la Vida de El Pangui).

La participación de muchas mujeres shuar en el activismo anti-minero durante estos años, tanto en organizaciones de mujeres como al lado de los hombres, les permitió hacerse más visibles en el terreno político y aumentar sus relaciones fuera de las comunidades. Por otro lado, uno de los rasgos que ha definido esta lucha es también lo que Casen (2013) denomina "esencialismo estratégico", siguiendo la terminología de Spivak, cuando analiza las movilizaciones sociales indígenas en Bolivia. Esta cualidad se refiere a la tendencia a utilizar la figura del indígena, y con ello, a reivindicar un ideal cultural que a menudo integra los estereotipos con los que se construye la identidad indígena, como forma de enfrentar un modelo neoliberal de desarrollo que privatiza y explota la tierra sin respetar las concepciones propias de las sociedades que allí habitan. En particular, en Ecuador, existen ciertos derechos colectivos en relación a la tierra reconocidos solo a la población indígena, lo que hace que en este contexto la etnicidad cumpla una función estratégica en la dimensión política, además de en la simbólica ${ }^{17}$.

El hecho de que la resistencia anti-minera haga uso de cierto esencialismo cultural para poder articular con más éxito sus demandas, tiene también efectos en las formas de participación de las mujeres. Por un lado, las mujeres son tomadas como portavoces legítimas en la defensa de la naturaleza por el carácter femenino que tiene la misma tierra, la fertilidad y el cuidado, lo que nos lleva a valorar el carácter estratégico que también puede tener el esencialismo de género ${ }^{18}$. Por otro lado, aunque posiblemente en relación con esta cuestión, la resistencia femenina contra el extractivismo puede igualmente visibilizar a las mujeres sin constituir en la práctica un instrumento que transforme problemas reales de las mujeres, como su exclusión en los sistemas de propiedad, o la falta de control sobre aspectos relacionados con

17 Como ejemplo podemos mencionar el caso de la Comunidad Amazónica de Acción Social Cordillera del Cóndor (CASCOMI), una organización de la zona que, a pesar de estar formada mayoritariamente por pequeños agricultores y ganaderos mestizos, su estrategia de resistencia incluyó la reivindicación de reconocimiento como comunidad indígena.

18 Recojo parte del comunicado prensa de las "Mujeres amazónicas en movilización por la vida", del 12 de octubre de 2013 en Puyo, al inicio de la marcha hasta Quito, que realizaron para protestar contra la explotación del Yasuní: "Como mujeres sentimos desde la profundidad de nuestros vientres las amenazas del extractivismo y consideramos de carácter urgente abrir el debate frente a la coyuntura que se ha generado a partir del tema Yasuní-ITT y salir en defensa de nuestra madre nutriente que pare, cría y ampara a todos sus hijos sin mirar acciones, etnias ni clases sociales. A una madre no se la explota ni se le extraen de sus entrañas el petróleo o el carbón para provecho de nadie, a ella se la respeta y se la ama por el simple hecho de haber provenido de su cuerpo. Estos actos simbolizan una mutilación de sus partes, equivalen a un matricidio". 
su propia sexualidad. De ahí que sea necesario entender la complejidad de los procesos de transformación que atraviesan las sociedades amazónicas afectadas por la industria extractiva.

Lo cierto es que en la actualidad, aunque la resistencia en el área sigue organizada $^{19}$, el Frente de Mujeres en Defensa de la Vida de El Pangui ya no funciona como tal y las acciones de protesta de las mujeres han ido disminuyendo, debido a cuestiones como la dura represión que sufrieron las y los activistas ${ }^{20}$ o el gran avance del Proyecto Mirador, cuyos trabajos en la zona se han incrementado, pues la empresa planea iniciar aproximadamente en 2018 la extracción de cobre, en cantidades que podrían llegar hasta las 60.000 toneladas de roca al día. Algunas de las mujeres entrevistadas hablan del asesinato en diciembre de 2014 del líder shuar José Tendet$\mathrm{za}^{21}$, conocido opositor a la minería, como un intento de silenciar las protestas antimineras. Una de sus hermanas relaciona la pérdida de fuerza de las protestas con este hecho, que causó una gran conmoción en las comunidades shuar: "Desde entonces menos protestan, de aquí ya no protesta nadie, de la comunidad nadie está contra la empresa. Mis hermanos ya dejaron, puede venir otra muerte".

Pero, sin duda, el temor más extendido en toda el área de impacto directo del Proyecto Mirador es el miedo al desalojo. La creciente expulsión de familias de sus tierras $^{22}$, unida a las dificultades percibidas por las mujeres al imaginar la vida fuera de sus comunidades, sin acceso al territorio ancestral, hace que prevalezca una generalizada visión negativa con respecto a su futuro.

Aquí estando en la finca uno se siembra de todo, en cambio en la ciudad, qué voy a sembrar, qué voy a comer, allí en la ciudad para comprar un maduro es dinero, si tengo ganas de chonta todo es con plata en la ciudad y aquí yo misma voy a la finca, cojo, miro, traigo, como, no tengo que estar comprando, en la ciudad es todo dinero. ¿Qué futuro va a haber? Ahí si ya va a ser duro (mujer shuar, 27 años, Yanua Kim).

Con independencia de que la mujer haya estado a favor o en contra de la minería a gran escala con anterioridad, la amenaza de reubicación y pérdida definitiva de los recursos que han garantizado siempre la supervivencia del pueblo shuar es vivida con tristeza y de forma contradictoria, pues viola los derechos colectivos de la población indígena de Ecuador recogidos por la Constitución, de habitar y hacer uso de sus territorios ancestrales ${ }^{23}$. En un momento de intensa transformación cultural para las sociedades shuar de Zamora Chinchipe, la contaminación y pérdida de sus tierras constituye para las mujeres de las comunidades (con escasa preparación para

19 CASCOMI es en la actualidad la organización que dirige principalmente la resistencia contra el Proyecto Mirador.

20 En los años de mayor actividad fueron acusados y arrestados tanto el dirigente del Comité en Defensa de la Vida, la Salud y la Naturaleza como la dirigente del Frente de Mujeres en Defensa de la Vida de El Pangui.

21 Información publicada en El Universo. "Mujeres Shuar piden investigar muerte de líder indígena José Tendetza”. El Universo, diciembre de 2014.

22 Los datos más precisos sobre desalojos forzosos en la zona en los últimos años los da el Colectivo de Investigación y Acción Psicosocial Ecuador, junto con Acción Ecológica. En el informe que publican en 2017 contabilizan 136 personas desalojadas, de las que ninguna de las que habitaban en Tundayme habría sido reubicada (2017: 91-94).

23 Artículos 60 y 57 (numerales 4, 5 y 6) de la Constitución de la República del Ecuador. 
el trabajo fuera de ellas, y en general, con responsabilidades familiares) el fin de la posibilidad de sobrevivir con sus propios medios, sin depender de un mercado laboral dominado por las transnacionales.

\section{Conclusiones}

En este artículo he querido explorar las percepciones y respuestas de las mujeres shuar afectadas por el Proyecto Mirador, el proyecto de minería a gran escala más importante de Ecuador, situado en la provincia de Zamora Chinchipe. Al hacerlo, se han destacado algunas cuestiones que reflejan la especial complejidad de su situación.

En primer lugar, analizamos un discurso que integra algunos valores que se contraponen a aquellos que legitimarían un desarrollo basado en una ética individualista y del trabajo desde el punto de vista occidental. Las mujeres shuar entrevistadas defienden principalmente un ideal de bienestar de la naturaleza que les permita mantener con normalidad los trabajos de alimentación y cuidado de la familia, así como la venta de los productos de su huerta en los mercados. Aunque algunas mujeres shuar optan por salir de las comunidades y acceden a trabajos remunerados con los que adquieren una mayor autonomía, son muchas también las que regresan a sus comunidades de origen con experiencias de discriminación, estrés o violencia, buscando un entorno más saludable con el que poder sentirse identificadas. Su rechazo general a un modelo que entiende la naturaleza meramente como un recurso económico forma parte a su vez de un discurso que reivindica la importancia del papel femenino en la cultura tradicional shuar, a pesar de mostrar satisfacción con las transformaciones que han vivido en las últimas décadas en el plano de la afectividad/sexualidad, como la pérdida de vigencia de la poligamia o de los matrimonios concertados de las niñas con hombres mayores.

En segundo lugar, al analizar los cambios en las relaciones de género hemos visto que estos también pueden verse afectados por las nuevas pautas adquiridas en las comunidades desde que se da en ellas una mayor dependencia del mercado de trabajo. A su vez, el impacto social producido por el extractivismo podría presentarse acentuado por la mayor fragilidad de las familias. Como reflexión al estudiar este aspecto se señalaba la especial vulnerabilidad de las mujeres shuar pues, si bien pueden en muchos casos acceder a una fuente de ingresos propia gracias a los trabajos ofertados por la industria minera a las mujeres, su posición dentro de las comunidades las somete a una considerable presión social que limita su autonomía, y cuando salen también se enfrentan generalmente a una mayor discriminación. Por otro lado, la mayoría de ellas rechaza las duras condiciones del trabajo en la empresa, en la que se emplean principalmente por necesidad.

En tercer lugar, hemos de considerar que la búsqueda de estas mujeres de un espacio comunitario donde poder vivir de la naturaleza, sin tener que recurrir a la minería, se expresa en medio de un proceso de profunda aculturación que tiende a quitar valor a las actividades femeninas que antiguamente otorgaban estatus social a las mujeres shuar. Lo cierto es que la forma particular en que se dan los impactos del desarrollo en las mujeres indígenas sigue haciendo necesario emplear un enfoque ecofeminista (Murphy, 2013), y entender que las categorías que utilizamos para hablar del género, el desarrollo, la identidad o la sostenibilidad, deben ampliarse 
para poder abarcar la complejidad que implica un profundo proceso de cambio en un escenario de "lucha de sentidos", en palabras de Guerrero (2011).

Para finalizar, se ha destacado el generalizado posicionamiento de las mujeres entrevistadas en contra de la minería a gran escala, en un momento en el que se aceleran los trabajos de la empresa y en el que las mujeres perciben como amenaza muy directa la contaminación y el desalojo de sus hogares. Al analizar la participación femenina en los procesos de resistencia en la zona, se señalan algunas características peculiares del liderazgo femenino shuar, como el hecho de que tenga como objetivo la defensa de la naturaleza, una reivindicación legítima para las mujeres shuar desde su cultura, o la posibilidad de que a través de estas acciones puedan conseguir una mayor visibilización o acceder a nuevos espacios de poder. Ambos aspectos se relacionan a su vez con las dinámicas contradictorias que afectan de forma general a las mujeres indígenas en espacios de gran biodiversidad invadidos por la industria extractivista. Como aquí hemos visto, es posible apreciar un aumento de las expectativas e intercambios de las mujeres fuera de su cultura de origen que puede brindarles nuevas oportunidades, en coexistencia con una pérdida de autonomía y estatus vinculada con su mayor vulnerabilidad socioeconómica (en un contexto con una grave amenaza territorial) y con la pérdida de los valores y símbolos tradicionales de la cultura shuar.

A su vez, aquí se ha relativizado el impacto que puede tener este aumento de visibilidad social en la transformación de las identidades de género, o en la mayor autonomía femenina. Para sostener esta afirmación he señalado la importancia que tiene la articulación estratégica de la etnicidad dentro del activismo anti-minero en la zona, vinculándola además con el esencialismo de género en el que también se apoya al asimilar la naturaleza a lo femenino. No obstante, esta cuestión expresa la especial complejidad que caracteriza el estudio de las sociedades indígenas en la contemporaneidad $^{24}$ y exige una mirada que transcienda las categorías que desde el discurso científico empleamos para definir el desarrollo. Esto no significa que las mujeres que se han desarrollado en el contexto de sociedades indígenas no deseen alcanzar muchos de los estándares de equidad de género con los que trabaja el feminismo occidental. Lo que se apunta más bien es que estos deseos se dan generalmente dentro de un esquema cultural en el que el concepto de bienestar también está atravesado por otras cuestiones que pueden no estar presentes en esquemas aparentemente más neutros.

\section{Referencias bibliográficas}

Barriga, Franklin (1986). Etnología ecuatoriana. II Shuaras. Quito: Editorial Santa Rita, IECE. Cabnal, Lorena (2010). "Acercamiento a la construcción de la propuesta de pensamiento epistémico de las mujeres indígenas feministas comunitarias de Abya-Yala”, en VV.AA.: Feminismos diversos: el feminismo comunitario. ACSUR-Las Segovias, 11-24.

24 Díaz de Rada analiza esta cuestión en profundidad en su artículo "La ironía de ser indígena y la imaginación del tiempo social", publicado en 2015 en la Revista de Antropología Social, Vol. 24. En su opinión, la antropología se ve obligada a analizar críticamente "la condición alócrona del sujeto construido por la antropología como objeto", al que tendemos a negar su contemporaneidad al situarlo en un tiempo mítico, como anteriormente se situaba al "indígena" en un "estado de naturaleza" que en última instancia favorecía su deslegitimación como agente político (Díaz de Rada, 2015: 441). 
Cardno (2014). “Actualización del Estudio de Impacto y Plan de Manejo Ambiental de la Fase de Explotación. Proyecto Minero de Cobre Mirador para la Ampliación a 60 kt por día". Ministerio del Ambiente, Ecuador.

Casen, Cécile (2013). "La figura del indígena como encarnación del pueblo boliviano: discusión en torno al esencialismo estratégico del Movimiento al Socialismo (MAS)", Rúbrica Contemporánea, Vol. 2, Núm. 3: 67-82.

Costales, Alfredo; de Costales, Piedad (2006). La Nación Shuar. Tomo I. Quito: Ediciones Abya-Yala.

Colectivo de Investigación y Acción Psicosocial, Ecuador (2017). La herida abierta del Cóndor. Quito: El Chasqui Ediciones.

Cuvi, María (2000). "Mujeres, género y feminismos: una mirada desde la otra orilla", en: CORDES y CEDIME. Mujer, participación y desarrollo. Quito: CORDES/CEDIME, 4575.

Del Campo, Esther (2012). “Democracia, política y políticas públicas: las mujeres indígenas como nuevos actores estratégicos en América Latina", en: Del Campo, Esther (Ed.) Mujeres indígenas en América Latina: politica y politicas públicas. Madrid: Fundamentos, 7-44.

Descola, Philippe (1996). La selva culta. Simbolismo y praxis en la ecología de los Achuar. Quito: Abya-Yala.

Díaz de Rada, Ángel (2015). "La ironía de ser indígena y la imaginación del tiempo social", Revista de Antropología Social, Vol. 24. Universidad Complutense de Madrid, 433-449.

Espinosa, María Fernanda (1998). "La Amazonía Ecuatoriana: Colonia Interna”, ICONOS, No. 5: 28-34.

Garcés, Alicia (2006). Relaciones de género en la Amazonía ecuatoriana. Estudios de caso en comunidades indigenas Achuar, Shuar y Kichua. Quito: Ediciones Abya-Yala.

Gargallo, Francesca (2014). Feminismos desde Abya-Yala. Ideas y proposiciones de las mujeres de 607 pueblos de nuestra América. Ciudad de México: Editorial Corte y Confección.

Giz (2011). "Pueblos indígenas en Ecuador. Documento de trabajo. Unidad Coordinadora Pueblos Indígenas en América Latina y el Caribe (KIVLAK/GIZ)". [En línea] Consultado el 6 de julio de 2015. URL: http://www.giz.de/expertise/downloads/giz2011es-laenderpapier-ecuador.pdf

Gudynas, Eduardo (2009). El mandato ecológico. Derechos de la Naturaleza y políticas ambientales en la nueva Constitución. Quito: Abya-Yala.

Guerrero, Patricio (2011). "Interculturalidad y plurinacionalidad, escenarios de lucha de sentidos: entre la usurpación y la insurgencia simbólica”, en: Kowii Maldonado, A. (Coord.) Interculturalidad y diversidad. Quito: UASB y CEN.

Hendricks, Janet W. (1996). "Poder y conocimiento: Discurso y transformación ideológica entre los Shuar", en: Santos, Fernando (Comp.) Globalización y cambio en la Amazonía indígena. Quito: FLACSO, Biblioteca Abya-Yala, 131-181.

Karsten, Rafael (2000). La vida y la cultura de los Shuar. Quito: Ediciones Abya-Yala.

León, Magdalena (2008). 'Después del 'desarrollo': 'el buen vivir' y las perspectivas feministas para otro modelo en América Latina”, Revista Umbrales, $\mathrm{N}^{\circ} 18: 36-44$.

Lu, Flora; Bilsborrow, Richard E.; Oña, Ana Isabel (2012). Modos de vivir y sobrevivir. Un estudio transcultural de cinco etnias en la Amazonia ecuatoriana. Quito: Ediciones Abya-Yala.

Mader, Elke (1999). Metamorfosis del poder: persona, mito y visión en la sociedad de Shuar y Achuar (Ecuador, Perú). Quito: Abya-Yala. 
Martínez-Alíer, Joan (2004). El ecologismo de los pobres. Conflictos ambientales y lenguajes de valoración. Barcelona: Icaria.

Méndez, Georgina (2009). "Miradas de género de las mujeres indígenas en Ecuador, Colombia y México", en: Pequeño, Andrea (Comp.) Participación y políticas de mujeres indígenas en América Latina. Quito: FLACSO, 53-71.

Mies, Maria y Shiva, Vandana (1997). Ecofeminismo: Teoría, crítica y perspectivas. Barcelona: Icaria.

Mies, Maria; Shiva, Vandana (1998). La praxis del ecofeminismo. Barcelona: Icaria.

Muratorio, Blanca (2000). "Identidades de mujeres indígenas y política de reproducción cultural en la Amazonía ecuatoriana”. En Guerrero, Andrés (Comp.) Etnicidades. Quito: FLACSO, Sede Ecuador, 235-266.

Murphy, Patrick D. (2013). "The ecofeminist subsistence perspective revisited in an age of land grabs and its representations in contemporary literature", en: Kerslake, Lorraine y Gifford, Terry (Coords.) Ecofeminismo/s: mujeres y naturaleza. Feminismo/s $n^{\circ} 22$, CEM, Universidad de Alicante, 205-224.

Poats, Susan (1999). "La dimensión de género en el manejo alternativo de conflictos socioambientales: una exploración preliminar", en: Ortiz-T., Pablo (Comp.) Comunidades y conflictos socioambientales: experiencias y desafios en América Latina. Quito: Ediciones Abya-Yala - COMUNIDEC, 141-153.

Ortiz-T., Pablo (1997). Globalización y conflictos socioambientales. Quito: Abya-Yala.

Paredes, Julieta (2008). Hilando fino desde el feminismo comunitario. México: Editorial El Rebozo.

Pellizzaro, Siro M., Martínez, Gracia, Tsere, Washíkiat y Katan, Tuntíak Patricio (2009). Mitos Shuar. Tomo I. Nunkui. Quito: Abya-Yala.

Puleo, Alicia H. (2011). Ecofeminismo para otro mundo posible. Madrid: Cátedra.

Rubestein, Steven (2005). "La conversión de los Shuar”, ICONOS, 22: 27-48.

Santos, Fernando (1996). "Hacia una antropología de lo contemporáneo", en: Santos, Fernando (Comp.) Globalización y cambio en la Amazonía indígena. Volumen I. Quito: Ediciones Abya-Yala, 7-45.

Shiva, Vandana (2006). Manifiesto para una Democracia de la Tierra: justicia, sostenibilidad y paz. Barcelona: Paidós Ibérica.

Tene, Carmen (2000). "Ruptura de la exclusión de mujeres indígenas", en: CORDES y CEDIME. Mujer, participación y desarrollo. Quito: CORDES/CEDIME, 199-224.

Villena, Sergio (2000). "Globalización y multiculturalidad. Pueblos indígenas y medio ambiente en la Amazonía”, Perfiles Latinoamericanos, 17: 95-122. 